\title{
Fluxo de sedimentos em suspensão nos rios da Amazônia
}

\author{
Naziano Filizola ${ }^{1}$ \& Jean Loup Guyot ${ }^{2}$
}

\begin{abstract}
Resumo ABacia Amazônica se estende por mais de $610^{6} \mathrm{~km}^{2}$, e aporta ao Oceano Atlântico um volume d'água da ordem de $6.60010^{9} \mathrm{~m}^{3}$.ano-1. Cerca de $50 \%$ da bacia corresponde aos terrenos pré-cambrianos dos escudos (Guianas e do Brasil Central), 12\% à cadeia andina e o restante corresponde à planície fluvial amazônica. Resultado da erosão dos Andes os sedimentos são transportados até o Oceano Atlântico através dos rios, principalmente. Estudos relativos a este tipo de estudo na Bacia Amazônica são raros, muitos dos quais concentrados na porção central da planície de inundação. Assim, o presente artigo apresenta resultados obtidos a partir de dados de campo, fruto de coletas realizadas em algumas estações de referência nos principais rios da bacia, bem como dados coletados em campanhas de campo realizadas em todo o contexto da região. A distribuição da Matéria Em Suspensão (MES) é descrita sob diferentes abordagens e condições hidrológicas em contraste com estudos anteriores que indicam diferentes valores de fluxo de MES. O regime hidrossedimentométrico dos principais tributários do Rio Amazonas é analisado de maneira cruzada, a partir de diferentes bases de dados disponíveis: campanhas de campo, estações de referência e redes nacionais de coleta de dados hidrometeorológicos. Esses diferentes tipos de abordagem vêm de fontes distintas, tanto de amostragem como de processamento das amostras, e também dos dados (Cálculos de fluxo). Os fluxos de sedimentos em suspensão advindos através dos rios oriundos dos escudos pré-cambrianos que compõem parte da morfoestrutura da Bacia Amazônica, até então pouco conhecidos, são apresentados. Por fim, o fluxo total de material em suspensão exportado pelo Amazonas é aqui estimado, a partir dessas bases, entre 600 e $80010^{6}$ t.ano ${ }^{-1}$.
\end{abstract}

Palavras-chave: sedimentos, hidrologia, Amazônia, rios.

\begin{abstract}
Suspended Sediment Flow in the Amazon Rivers. The Amazon River Basin extends over 6,1 million $\mathrm{km}^{2}$ and releases a fresh water volume of $6,60010^{9} \mathrm{~m}^{3} . \mathrm{ano}^{-1}$ to the Atlantic Ocean. About $50 \%$ of the area of the basin corresponds to the pre-Cambrian lands, the shields, $12 \%$ correspond to the Andean chain and the remain corresponds to the Amazonian plain. As a result of the Andean chain erosion, the transport of sediments at the Amazon basin until the Ocean, has in the rivers its main agent. The studies concerning this transport, have been rarefaied and most of them concentrated a long of the great central valley and sometimes, very rare, in some of its main tributaries. This study presents a vision of that subject since the level of the measurement sections at hydrometric stations until the continental scale. We evaluate the distribution of the total suspended sediment budget at the Amazonian Basin and some methods to calculate suspended sediment discharge as well. We also quantifying the contributions of the main sources of sediments, including the shields, until know inedit. Finally, we analyze distinct databases and evaluate the total contributions of the River Amazon in terms of annual flow of suspended sediments to the Atlantic Ocean, considering a new value, which resulted in a variation between 600 to 800 million tons.
\end{abstract}

Keywords: sediments, hidrologia, Amazon, rivers.

INTRODUÇÃO O rio e sua bacia de drenagem constituem uma unidade funcional para o ciclo da água e, assim também, um espaço integrador privilegiado para o estabelecimento de balanços ou ainda de validação de modelos de alteração e erosão. Portanto, os rios estão no coração do ciclo dos elementos, transportando para os oceanos a matéria sublevada dos continentes. Respondem muito rápido às condições do meio existente na superfície dos continentes, em alguns meses para o caso do transporte em solução, e em alguns anos para o caso do transporte em suspensão. Assim, são particularmente sensíveis a toda mudança, seja ela climática, ou então resultante de atividades humanas (antropismo). Numa rede hidrográfica, os rios exportam para os oceanos uma grande parte dos materiais incluídos no processo de erosão, tanto sob forma dissolvida como sob forma particulada. As partículas são colocadas em movimento sob a forma de Matéria Em Suspensão (MES) ou de transporte de fundo.

Aportes globais de MES aos oceanos e o papel das grandes bacias tropicais As grandes bacias fluviais tropicais representam $25 \%$ das terras emersas dos continentes e $57 \%$ da água doce disponível na superfície do

1 - Laboratório de Potamologia Amazônica, Universidade Federal do Amazonas, Manaus (AM), Brasil. E-mail: naziano.filizola@gmail.com

2 - Laboratoire des Mécanismes de Transfert en Géologie - LMTG, IRD/CNRS - Université de Toulouse, Toulouse, França. E-mail: jean-loup.guyot@ird.fr 
planeta. São assim, responsáveis por 50\% dos aportes sólidos, a Matéria Em Suspensão ou MES, e 38\% dos aportes em solução. Ainda, as maiores taxas de erosão são observadas nas bacias hidrográficas situadas na faixa tropical e particularmente naquelas que drenam cadeias de montanha ativas (Pinet \& Souriau 1988, Milliman \& Syvitzki 1992, Summerfield \& Hulton 1994), como os Andes, por exemplo. Os processos de erosão, de transporte e de sedimentação são sensíveis às mudanças climáticas globais (Julien 1995, Aalto et al. 2003). Neste contexto, o relevo e o clima são os principais motores da dinâmica de transferências de matéria na superfície da Terra. No caso das grandes bacias continentais, como é a Bacia Amazônica, a pressão antrópica - desmatamento, atividades agrícolas, exploração mineral e urbanização - feitas sem controle, ocorrendo já há alguns anos (Sternberg 1975, 1995), apresentam uma influência não negligenciável sobre o processo de erosão. Os resultados destas atividades humanas geram modificações na cobertura vegetal e nos solos que, a seu tempo, induzem modificações no regime climático (Shukla et al. 1990).

A importância da Bacia Amazônica Bacia Tropical por excelência, a Bacia Amazônica (Fig. 1), se estende por cerca de 6,1 milhões de $\mathrm{km}^{2}(5 \%$ das terras emersas continentais do globo), aporta ao oceano um volume de água de aproximadamente $6,6.10^{12} \mathrm{~m}^{3} . \mathrm{ano}^{-1}$, o que corresponde a cerca de $16 \%$ a $20 \%$ do total das águas doces continentais (Molinier et al. 1996). A Bacia Amazônica apresenta características climáticas e topográficas contrastantes. A parte oriental da bacia de drenagem é caracterizada por uma cadeia de montanhas ativa, os Andes, que correspondem a $12 \%$ da superfície total da região. Porém, aportam a quase totalidade da MES transportada pelos grandes rios Amazônicos na planície (Sioli 1950, 1975, Gibbs 1967, Meade et al. 1985, Guyot et al. 1994, Filizola 1999, Filizola 2003, Filizola \& Guyot, 2009). A vasta planície amazônica, onde áreas de sedimentação podem ser identificadas (Guyot 1993, Espurt et al. 2007), encontra-se rodeada por terrenos geologicamente muito antigos (Pré-Cambrianos), correspondentes aos escudos: Brasileiro e das Guianas. Englobando todas essas unidades morfoestruturais, o relevo varia desde o nível do mar até mais de 6.000 $\mathrm{m}$ de altitude, a pluviosidade varia de 100 até cerca de $5.000 \mathrm{~mm} \cdot \mathrm{ano}^{-1} \mathrm{e}$ a vegetação é, em grande parte, constituída por $70 \%$ de floresta tropical úmida.

Erosão e transporte de sedimentos na Bacia Amazônica O estudo dos fluxos sólidos da bacia Amazônica

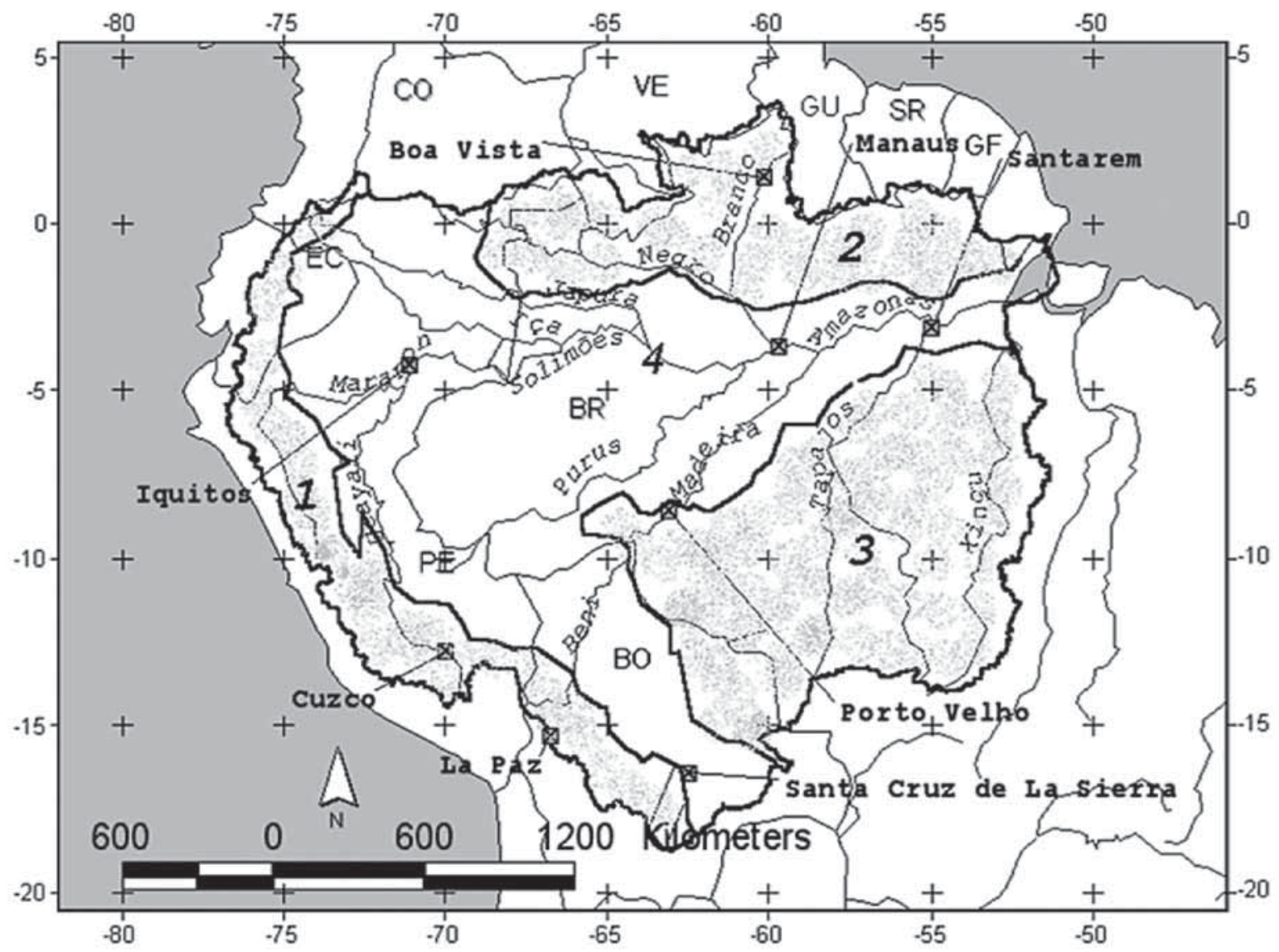

Figura 1 - A Bacia Amazônica com suas 4 principais unidades morfoestruturais (1 - Cadeia Andina; 2 - Escudo das Guianas; 3 - Escudo Brasileiro e 4 - Planície fluvial) e seus principais cursos d'água. Fonte: modificado de Filizola (2003). 
vem desde os anos 1950/1960. Esses estudos mostraram ser os Andes a principal fonte de sedimentos para os rios Amazônicos (Sioli 1964, 1984, Gibbs 1967, Guyot 1993, Filizola 1999, Filizola \& Guyot 2009). Na região da planície Amazônica, processos de estocagem (sedimentação) e de ressuspensão já foram observados (Schmidt 1972). Tais fenômenos foram associados a mudanças no gradiente hidráulico ao curso do ciclo hidrológico (Meade 1985, 1988, Richey et al. 1986, Ferreira et al. 1988, Dunne et al. 1998). As estimativas do fluxo de MES exportadas pelo Amazonas aos Oceanos variaram no tempo, segundo os autores: $500.10^{6}$ ton. ano $^{-1}$ (Gibbs 1967), 800-900.106. ton.ano ${ }^{-1}$ (Meade et al. 1979), 1.100 a $1.300 .10^{6}$ ton.ano ${ }^{-1}$ (Meade et al. 1985, Richey et al. 1986, Meade 1988). Em 1999, Filizola propôs uma retomada do valor inicialmente calculado por Bordas et al. (1988) e Bordas (1991) como sendo $600.10^{6}$ ton. ano $^{-1}$. Esses valores foram revistos por Filizola (2003) e Filizola \& Guyot (2009) que ampliaram a faixa de variação para valores entre $600-800.10^{6}$ ton. ano $^{-1}$, utilizando vários modos de abordagem. Uma vez que esses valores foram confirmados por Guyot et al. (2005), esse parâmetro vem sendo acompanhado desde 2003 pela rede ORE-HYBAM, que no Brasil é gerenciada por uma base técnica instalada em Manaus-AM (Filizola et al. 2007).
OBJETIVOS Dentro do contexto acima colocado, este artigo apresenta uma síntese dos estudos de balanço de fluxo de MES na Bacia Amazônica, analisando e comparando resultados oriundos de 3 fontes distintas de dados (Fig. 2), gerados no escopo dos trabalhos da cooperação Brasil-França no período de 1995 a 2000 descritos em Filizola (2003) e em Filizola \& Guyot (2009).

\section{METODOLOGIA E MODO DE ABORDAGEM}

A abordagem aqui adotada para o balanço de MES retoma a de Filizola (2003) e Filizola \& Guyot (2009), utilizando-se de 3 fontes distintas de dados, sendo: i) amostragens detalhadas eventuais, realizadas ao menos 3 vezes ao ano através de campanhas de campo independentes, no período de 1995 a 1998, em estações das redes nacionais de hidrometria no Brasil e na Bolívia (Base de dados do Projeto HIBAM). Uma campanha extra por ano foi realizada de 1999 a 2000, cujos dados também foram incorporados. As campanhas foram realizadas sempre buscando o período de subida das águas, pico de cheia (Maio), descida das águas e pico de seca (Outubro/Novembro), tendo o hidrograma de estação de Óbidos como referência. Estas campanhas se deram em embarcações regionais onde as coletas eram filtradas após realização em profundidades distintas ao longo de uma seção transversal ao fluxo do curso d'água e em diferentes pontos (estações hidrométricas da ANA

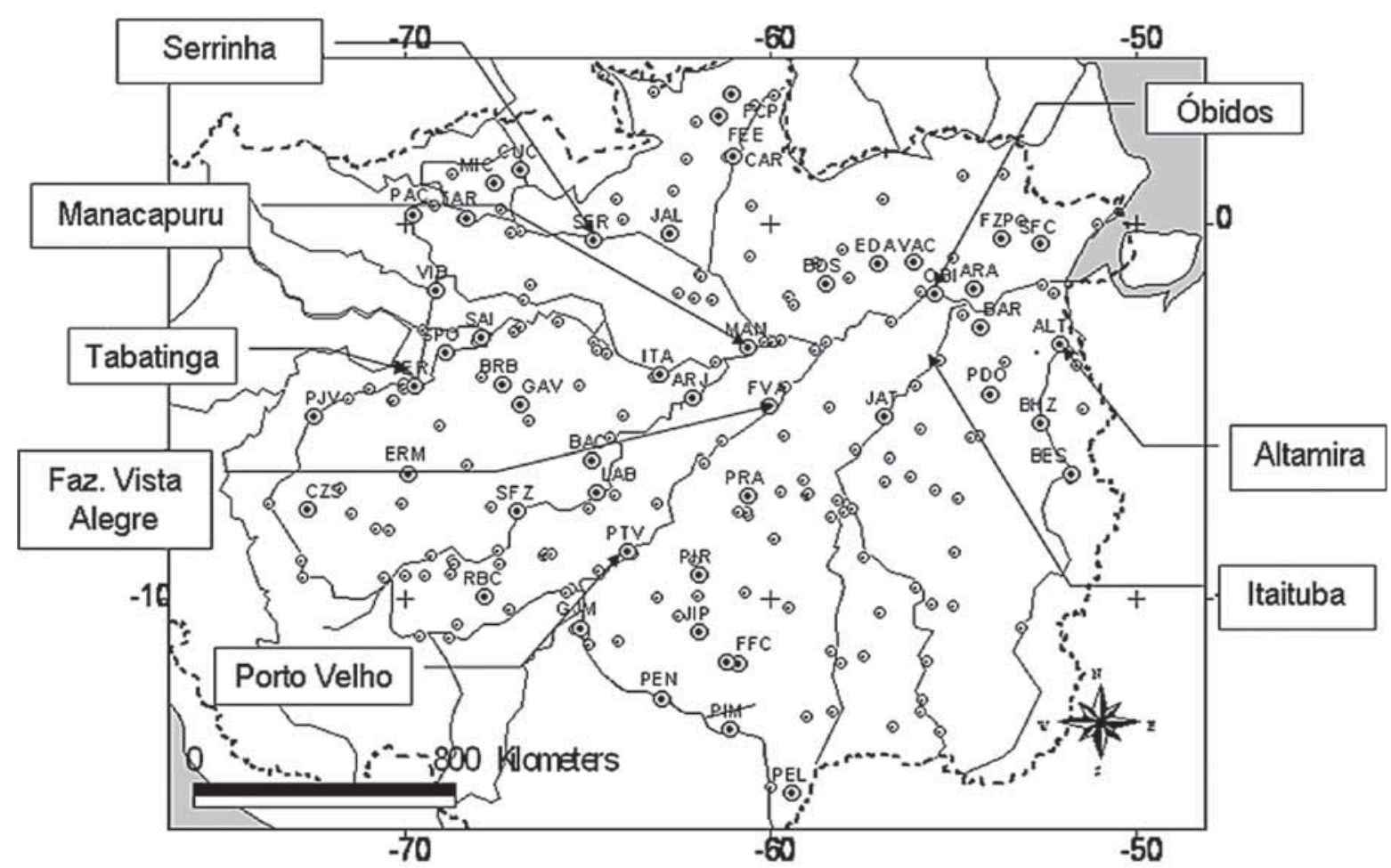

Figura 2 - Localização das estações de referência do Programa HIBAM sobre o mapa das estações hidrométricas da ANA na Amazônia. As estações com mnemônicos (3 letras) são estações onde a ANA faz coleta de MES a cada 3 meses. As estações cujos nomes completos estão indicados em retângulos são aquelas operadas pelo Programa ORE/HYBAM com amostras coletadas a cada 10 dias. Fonte: Modificado de Filizola (2003). 
- Agência Nacional de Águas) de um mesmo rio Amazônico (curso principal e os grandes tributários), em concomitância com medições de vazão; ii) amostragens realizadas desde 1995 a cada 10 dias por observadores contratados em pontos também coincidentes com as estações hidrométricas da rede nacional da ANA. Neste caso, somente foram coletadas amostras na superfície (Base da rede de estações de referência), sem medições de vazão. Os cálculos de vazão foram realizados com dados obtidos da base da ANA e iii) amostragens detalhadas realizadas pelas redes de estações hidrométricas nacionais (Brasil e Bolívia), notadamente pela rede da ANA, no caso brasileiro, através de campanhas de campo regulares realizadas em média a cada 3 meses em concomitância com medições de vazão realizadas pelos operadores da rede hidrométrica. Cada um dos 3 procedimentos deu origem a uma base de dados diferente. A coletada dos dados que formaram cada uma das bases utilizadas implicou num procedimento metodológico diferenciado que será descrito mais adiante individualmente. Para o cálculo do balanço de fluxos, utilizou-se a equação do balanço de massa, ou seja:

$$
(\mathrm{QSa}+\mathrm{QSb}+\ldots+\mathrm{QSn})-\mathrm{QSx}=\boldsymbol{\varepsilon}
$$

Para o caso da equação (1), QSa é o fluxo de MES medido numa estação "a" a montante do ponto "x" num determinado curso d'água A. QSb é o fluxo de MES medido numa estação "b" a montante do mesmo ponto "x", porém num curso d'água B. QSn é o fluxo de MES medido numa estação " $n$ " a montante do mesmo ponto "x", porém no enésimo curso d'água. QSx é o fluxo de MES na estação, ou ponto "x" num curso d'água $\mathrm{X}$ a jusante dos demais, sendo deles uma prolongação natural rumo à foz. $\mathcal{E}$ é um valor positivo ou negativo conforme o efeito havido no trecho considerado entre os pontos "a", "b", "n" e "x" (a área de contribuição hidrológica). Assim, para a ocorrência de sedimentação no trecho citado, $\sum$ será positivo e para ressuspensão ou produção de MES no trecho, $\Sigma$ será negativo.

Os valores do balanço foram tomados como representativos das respectivas "áreas de contribuição hidrológica", georreferenciadas e cartografadas a montante de cada estação hidrométrica utilizada. Apesar de saber-se que os fenômenos acontecem verdadeiramente ao longo do canal do curso d'água e da contígua planície de inundação, adotou-se tal procedimento apenas para facilitar uma representação espacial geral.

A Base de dados do Projeto HIBAM A primeira fonte de dados analisada é constituída dos resultados obtidos através de 8 campanhas de campo realizadas no período de 1995 a 1998 ao longo dos grandes rios Amazônicos: Negro, Solimões, Japurá, Javari, Iça, Juruá, Jutaí, Purus, Madeira, Tapajós, Trombetas e Xingu (Fig. 3). Essa base se encontra incorporada à base

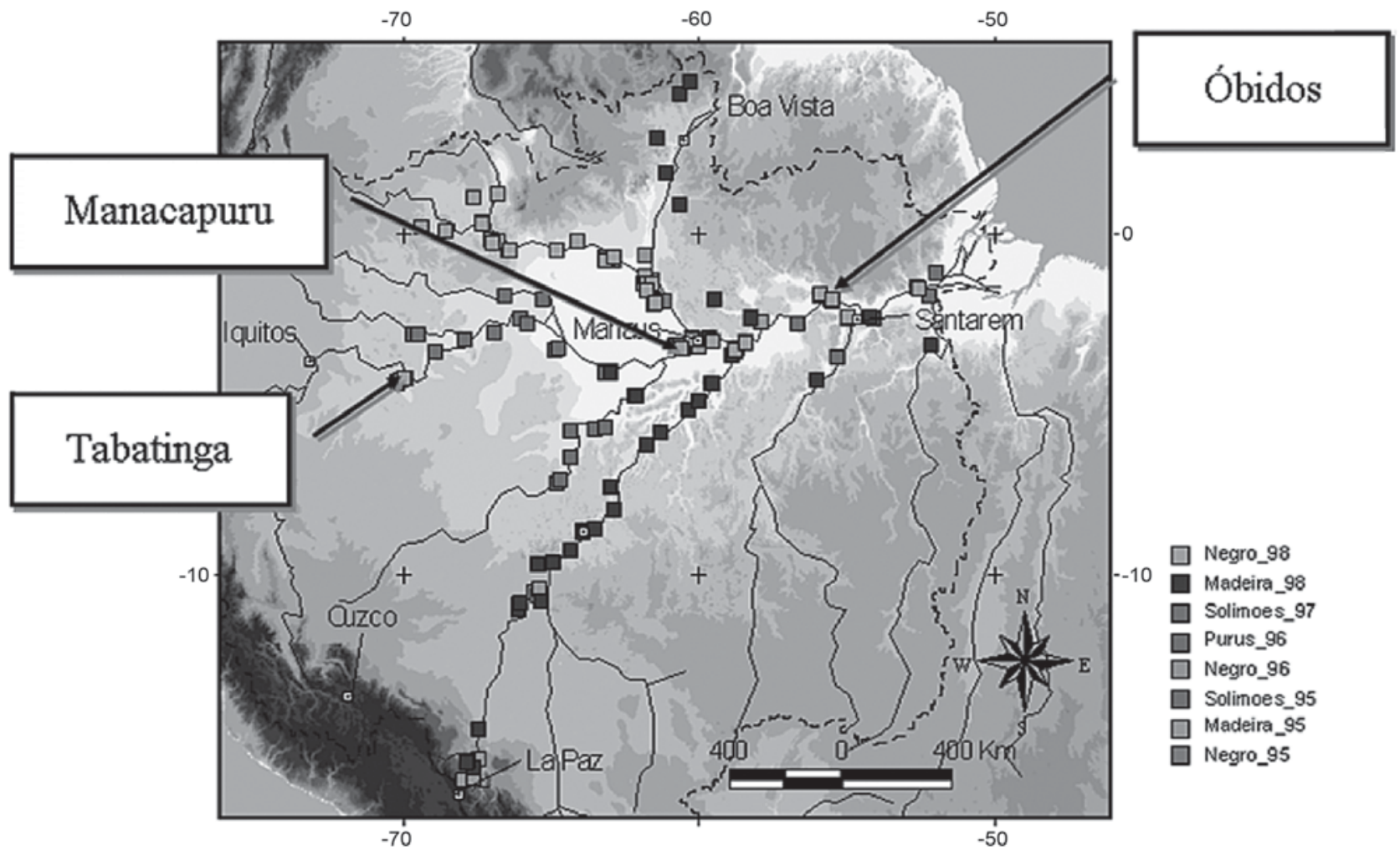

Figura 3 - Ilustração de alguns dos trechos de rios percorridos pelas campanhas HIBAM durante o periodo de 1995 a 1998 nos principais rios da Bacia Amazônica, com os quadrados indicando os pontos de coleta de amostras. Fonte: Filizola (2003). 
do atual Programa ORE/HYBAM, disponível em www.ore-hybam.org. A metodologia de coleta utilizou de amostrador pontual de grande volume desenvolvido especialmente para o estudo (Filizola \& Guyot 2004). Com tal equipamento se tornou possível realizar amostragens de até $12 \mathrm{~L}$ de água a várias profundidades, na seção molhada, indo desde a superfície até bem próximo do fundo, sob a supervisão de um Acoustic Doppler Currente Profiler (RD Instruments 1989), que também media de modo contínuo a vazão do rio na mesma seção. Além disso, parâmetros físico-químicos básicos de controle ( $\mathrm{pH}$, Temperatura, condutividade elétrica e turbidez) foram tomados a cada ponto amostrado. Seguindo este procedimento, foram coletadas 679 amostras pontuais de água para MES em 39 localidades distintas, nas quais foram também realizadas 89 medições de vazão no período acima citado, em diferentes épocas do ciclo hidrológico. Para o cálculo de fluxo de MES diária utilizou-se da relação onde $\mathrm{QS}=\mathrm{f}(\mathrm{Q})$, o que implica dizer que, com tal metodologia, ambos, fluxo de MES e vazão variam do mesmo modo na seção hidrométrica. Tal metodologia permitiu um detalhamento do que se passava nas seções em determinado momento do ciclo hidrológico (período de realização das campanhas), mas não permitiu uma análise mais detalhada do processo de fluxo em tempo mais curto (escala mensal), para o caso de a relação $\mathrm{QS}=\mathrm{f}(\mathrm{Q})$ não for válida. Para tal se fez necessário uma amostragem com maior frequência o que, por questões de logística amostral, impôs negligenciar o detalhamento da seção em função do melhor conhecimento do processo de fluxo no tempo.

Estações de alta frequência amostral Em face da necessidade acima exposta, numa segunda abordagem, construiu-se uma rede de estações de referência sobreposta à rede da ANA, aproveitando a infraestrutura já existente em: Serrinha no Rio Negro; Óbidos no Rio Amazonas; Manacapuru e Tabatinga no Rio Solimões; Fazenda Vista Alegre no Rio Madeira; Itaituba no Rio Tapajós e Altamira no Rio Xingu. Tais estações operaram do ano de 1995 até o ano 2000. Nessas localidades foram coletadas amostras de água, a $500 \mathrm{ml}$ de volume, para determinação de MES, com uma frequência amostral de 10 dias. Porém, essas amostras foram tomadas sempre à superfície. Um trabalho de calibração efetuado por Filizola (2003) correlacionou essas amostras, tomadas em superfície $\left([\mathrm{MES}]_{\text {sup }}\right)$, com as da seção total $\left([\mathrm{MES}]_{\mathrm{tot}}\right)$, obtidas durante as campanhas de campo. Naquelas campanhas, já citadas, os levantamentos pontuais na seção total foram realizados em maior detalhe em diversas verticais em profundidade e também em superfície conseguindo, assim, obter equações do tipo $[\mathrm{MES}]_{\text {tot }}=\mathrm{f}[\mathrm{MES}]_{\text {sup }}$, permitindo realizar uma correção das amostras superficiais. Esse tipo de relação implica num bom nível de conhecimento da distribuição dos sedimentos na seção. Este tópico foi avaliado por alguns autores (Filizola et al. 2009, Bouchez et al. 2011), porém estudos de maior detalhe estão sendo realizados pela equipe ORE/HYBAM, baseada atualmente em Manaus - AM. No total, para as estações de alta frequência amostral, foram analisados os resultados de 883 amostras. Esse esforço foi realizado em função de resultados anteriores mostrarem inconsistências com os valores listados na literatura produzida por outros grupos, até o início dos anos 90 . Os dados foram analisados em função da vazão média diária calculada a partir das informações das campanhas de campo complementadas pelos dados de vazão (apenas) da ANA. A existência de redes nacionais de coletada de dados e os resultados por ela produzidos para a Amazônia, ainda não haviam sido testadas com os dados acumulados após os trabalhos preliminares de Bordas et al. (1988) e Bordas (1991).

Estações das redes nacionais Assim, como terceiro modo de abordagem inclui-se, aqui, o uso de bases de dados oriunda das redes nacionais de amostragens de MES - Brasil e Bolívia; vinculadas às redes hidrométrica daqueles países. Do Brasil, onde a rede está sob a responsabilidade da Agência Nacional da Águas (ANA), foram utilizados resultados de mais de 9.000 amostras coletadas desde os anos 70 até o ano 2000 em uma rede de 60 estações distribuídas pela bacia no Brasil. Na Bolívia utilizou-se da base de dados PHICAB (IRD, SENAMHI, UMSA), hoje sob a responsabilidade do Serviço Nacional de Hidrologia e Meteorologia da Bolívia (SENAMHI), com mais de 27.100 amostras realizadas, descritas em Filizola \& Guyot (2009). Neste trabalho, no entanto, serão descritos, para fins de comparação, apenas dados das principais estações brasileiras, onde as operações foram coincidentes com aquelas dos demais modos de abordagem já explanados anteriormente. Para o caso das amostras da ANA, as mesmas foram coletadas, em sua maioria, utilizando método de integração vertical por igual velocidade de trânsito, fazendo uso de amostradores da série americana (Carvalho et al. 2000). No caso da Bolívia, foram utilizados amostradores pontuais de pequeno volume e a vazão foi medida com o uso de molinete hidrométrico, metodologia tradicional recomendada pela Organização Mundial de Meteorologia. No Brasil a maior parte das amostras foram coletadas pela CPRM (Serviço Geológico do Brasil) através de suas superintendências e escritórios nos estados da região. Após uma análise prévia e básica de consistência, os dados passaram a compor a base de dados da ANA, accessível on-line pelo site da Agência (www.ana.gov.br). O processamento das amostras em laboratório seguiu, no caso das amostras das campanhas e das estações da rede de referência, procedimento da rede GEMS/Water (PNUMA), que consiste da pré-pesagem (PI) de filtros de acetato de celulose de malha $0,45 \mu \mathrm{m}$ em balança digital (mínimo 2 algarismos significativo), filtração vertical com auxílio de bomba de vácuo, em rampa horizontal composta de 6 unidades de filtração de PVC com controle de filtrado, secagem em estufa a $110^{\circ} \mathrm{C}$ por uma hora e uma nova pesagem $(\mathrm{PF})$, para que depois se pudesse calcular o Peso Total da MES por diferença e relativo ao volume filtrado. Em seguida, calcular em mg.l-1 para, finalmente, multiplicar 
este valor de MES pela vazão média do dia e por um fator de conversão $(0,0864)$ obter-se o fluxo sólido instantâneo ou QS dado em toneladas por dia (ton.dia ${ }^{-1}$ ).

\section{RESULTADOS E DISCUSSÃO}

As campanhas de campo HIBAM Em vista da ausência de campanhas de campo do Programa HIBAM em todos os períodos do ciclo hidrológico, o valor de QS médio anual para o período de 1995 a 1998 foi calculado a partir das relações $\mathrm{QS}=\mathrm{f}(\mathrm{Q})$, onde $\mathrm{Q}$ é a vazão. Essas relações foram, então, elaboradas para cada estação de amostragem com dados HIBAM e com o auxílio das séries de dados de vazão fornecidos pela ANA. Os resultados permitiram propor uma primeira avaliação geral dos fluxos de MES nas principais estações de controle, assim como do fluxo ao Oceano Atlântico (Tab. 1). Também, os mesmos resultados, apesar de pouco dispersos no interior de um ciclo hidrológico, permitem uma avaliação de sua evolução temporal (Fig. 4). Por estes resultados e utilizando-se da equação (1), se pode avaliar que em março, no início da subida das águas, o fluxo de MES do Rio Madeira é superior ao do Rio Solimões e a tendência é de sedimentação (-23\%) entre Manacapuru e Óbidos. O valor de QS é próximo de $3.10^{6}$ ton.dia ${ }^{-1}$. No mês de abril, a subida das águas continua, os rios Solimões e Madeira contribuem praticamente com o mesmo fluxo de MES e a tendência de deposição (sedimentação) se amplia (-64\%), para valores da mesma ordem de grandeza do QS observado para o mês de março. No mês de maio, o Rio Amazonas em Óbidos chega a seu nível máximo. Neste período o Rio Solimões apresenta uma participação mais forte no fluxo sólido em Óbidos do que aquele do Rio Madeira. A tendência de deposição continua (-26\%) com um QS comparável àquele observado em março e abril. Em junho, a descarga líquida em Óbidos começa a diminuir e o fluxo sedimentar cai pela metade $\left(1,6 \cdot 10^{6}\right.$ ton. dia $\left.^{-1}\right)$. O essencial dos aportes sólidos vem do Rio Solimões. A tendência se inverte com o aumento da descarga sólida em Óbidos (+26\%). Em julho, a descida das águas continua em Óbidos e o Rio Solimões continua a fornecer o essencial da descarga sólida, da mesma ordem de grandeza que em junho. Os aportes particulados são curiosamente mais importantes (3 vezes maiores) no Rio Negro que sobre o Rio Madeira nesta época do ano, que se caracteriza, também, pela continuação do aumento do fluxo sedimentar em Óbidos (+56\%). Esse fenômeno é tradicionalmente atribuído à ressuspensão de MES depositado ao curso dos meses precedentes, porém é igualmente possível que uma boa parte das MES seja de origem orgânica. Nenhuma campanha HIBAM foi realizada em agosto e setembro nos anos aqui considerados. Nas campanhas realizadas em Outubro (1996 e 1998), em período de descida das águas, percebe-se muitas semelhanças com o fluxo sedimentar observado em Óbidos, da ordem de 300 a $40010^{3}$ ton.dia ${ }^{-1}$. Papel preponderante tem o Rio Solimões (QS de 74 a $85 \%$ do total em Óbidos). Neste período, também, o QS do Rio
Tabela 1 - Estimativa de fluxo sólido a partir de amostragem realizada durante as campanhas HIBAM, de 1995 a 1998. Também, para o mesmo periodo, o cálculo da vazão média anual e o percentual de representatividade de cada estação em relação aos valores obtidos para a estação de Óbidos (última estação com medição regular de vazão no Rio Amazonas, antes deste alcançar o oceano). Fonte: Filizola (2003).

\begin{tabular}{lcc}
\hline & $\begin{array}{c}\text { Vazão } \\
\left(\mathrm{m}^{3} \cdot \mathrm{s}^{-1}\right)\end{array}$ & $\begin{array}{c}\text { Fluxo sólido } \\
\left(10^{6} \text { ton.ano }\right.\end{array}$
\end{tabular}

Negro é superior ao do Rio Madeira. No entanto, a tendência para Outubro de 1996 foi de deposição (-27\%), enquanto que para o mesmo mês no ano de 1998 a tendência foi de um forte aumento $(+46 \%)$. Enfim, para o mês de novembro (já que nenhuma campanha foi realizada dos meses de dezembro a fevereiro), tem-se o mês de águas mais baixas. Esse mês é marcado pelo impacto do Rio Solimões que explica $75 \%$ da vazão

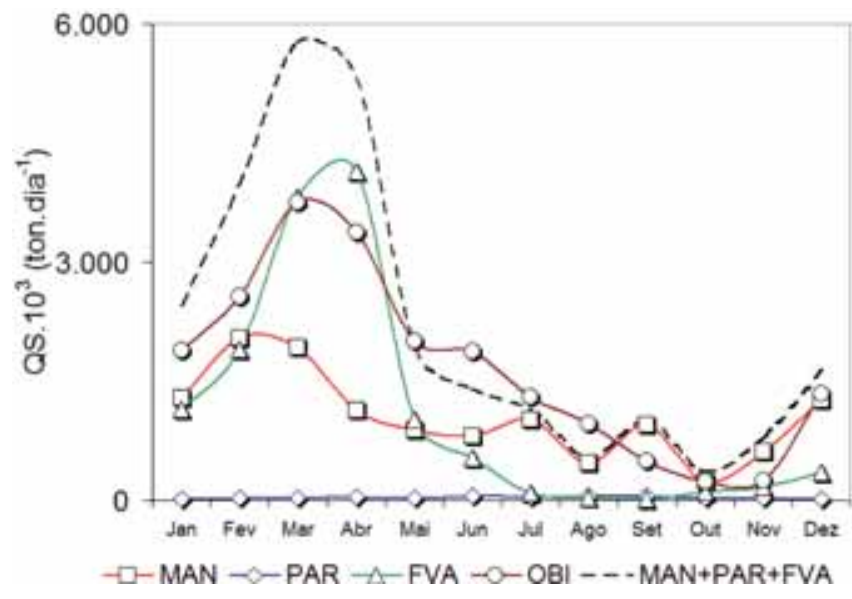

Figura 4 - "Hidrossedimentogramas" sobrepostos em escala mensal para os principais tributários do Rio Amazonas (MAN - Rio Solimões em Manacapuru; PAR - Rio Negro em Paricatuba; FVA - Rio Madeira em Faz. Vista Alegre e OBI - Óbidos no Rio Amazonas). Gráfico construido com dados compilados na escala mensal de www.ore-hybam.org, gerados a partir de amostragens de superficie, de alta frequência $(10 \mathrm{em}$ 10 dias), corrigidas por equações que relacionam $[M E S]_{t o t}=f[M E S]_{s u^{\prime}}$ de Filizola (2003). 
e $97 \%$ do fluxo sedimentar total observado em Óbidos. Nesta época do ano, o fluxo particulado, que é da mesma ordem de grandeza que em outubro, apresenta grande déficit (-108\%), representando uma forte sedimentação na porção do baixo Amazonas. Fenômenos de histerese ou "curvas em laço" para a relação QS = $f(Q)$ foram identificados para os rios de origem Andina, porém bem menos marcados nas estações localizadas sobre os escudos. Também, em relação ao Rio Madeira, foi possível verificar que o mesmo se individualiza por um comportamento diferenciado, segundo a estação do ano, ou seja: comportamento do tipo Andino, ou "rio de águas brancas" (histeresis) em época de águas altas e do tipo "rios de águas claras" (sem histeresis) em época de águas baixas.

Assim, no global médio interanual, pelos resultados da tabela 1, percebe-se que o Rio Solimões fornece ao Amazonas $62 \%$ da carga líquida e $54 \%$ da carga de matéria particulada em suspensão. O Rio Madeira, o segundo maior rio Andino da bacia, contribui com $17 \%$ da carga líquida e $45 \%$ da carga sólida. A estação de referência de Óbidos, última estação de controle hidrológico sem forte influência da maré, é aqui tomada como representativa dos aportes médios interanuais do Rio Amazonas ao Oceano Atlântico e apresenta um fluxo de MES da ordem de $71510^{6}$ ton.ano ${ }^{-1}$. Esse valor corresponde a um aumento aproximado de $10010^{6}$ ton. $\mathrm{ano}^{-1} \mathrm{em}$ relação às estimativas de Gibbs (1967), Meade et al. (1979), Filizola (1999) e praticamente a metade do valor proposto por Meade et al. (1985). Enfim, a soma dos fluxos dos Rios Solimões, Negro e Madeira teve como resultado $82610^{6}$ ton.ano ${ }^{-1}$. A diferença entre o observado e o calculado corresponde a uma estocagem média anual de pouco mais de $10010^{6}$ ton.ano $^{-1}$ de material sedimentar, dentro da bacia Amazônica, no trecho entre Manacapuru e Óbidos. Este valor é semelhante ao resultado de Meade et al. (1985).

As estações de referência HIBAM A rede de estações de referência HIBAM foi criada visando cobrir eventuais lacunas no ciclo hidrológico, buscando valorizar mais a influência da sazonalidade, normalmente não bem demarcada pelos estudos anteriores. Assim, o procedimento evidenciou diversos fenômenos. Porém, para efeito de comparação com os resultados acima apresentados, serão aqui destacados os dados para as mesmas estações (localidades e rios). Como as estações da rede de referência forneceram originalmente dados de superfície, estes foram corrigidos por equações que relacionam o fluxo de MES em superfície com o fluxo de MES total em cada seção, tomadas de Filizola (2003), desta forma puderam ser obtidos dados médios mensais de fluxo resumidos na tabela 2 , para a escala anual. Os dados das estações de referência, hoje incorporados à base www.ore-hybam.org, permitem conhecer de forma mais fina, na escala mensal, o regime hidrossedimentar do Rio Amazonas e de seus principais tributários (Fig. 4). Para o caso da estação de Paricatuba no Rio Negro, os dados de vazão utilizados foram obtidos pela diferença entre as descargas médias diárias entre as estações de Jatuarana (Rio Amazonas) adicionada com a estação do Careiro (Paraná do Careiro, Rio Amazonas), a jusante de Manaus, e a de Manacapuru (Rio Solimões), a montante. Os resultados gerais mostram que para os rios Andinos, o fluxo máximo de MES acontece antes do pico de cheia, com uma defasagem mínima para o caso do Rio Madeira (1 mês) e mais importante para o caso dos Rios Solimões e Amazonas (3 meses). De um modo geral para os rios Madeira, Solimões e Amazonas os picos de máxima de QS acontecem de fevereiro a abril e os valores mínimos de setembro a novembro. Para o Rio Negro o pico de máxima ocorre por volta do mês de junho e os menores valores por volta do mês de janeiro. Vale notar que, em média, no mês de setembro o fluxo sólido do Negro supera o do Madeira por um fator de aproximadamente 5, porém os valores são sempre muito baixos. Assim, também são bastante baixos e muito semelhantes aos do Rio Negro os fluxos encontrados para os rios Xingu e Tapajós, com base em dados obtidos das estações de referência neles instaladas (Altamira e Itaituba, respectivamente). Esses dois últimos rios têm, portanto, pouca influência no cômputo geral dos fluxos de MES do Rio Amazonas ao oceano. Os aportes de QS observados em Manacapuru são superiores aos de Óbidos de setembro a novembro e os do rio Madeira ultrapassam os de Óbidos entre março e abril. O QS máximo acontece em Manacapuru, em fevereiro, e, entre março a abril, no Madeira (Fazenda Vista Alegre). A estação de Óbidos apresenta seu pico de QS em março. A curva hidrossedimentológica em escala mensal, resultante da soma dos aportes dos 3 tributários considerados (Fig. 4), coincide grosseiramente com a curva observada para Óbidos de maio a novembro. No entanto, no período de águas altas a soma dos tributários é largamente superior aos valores

Tabela 2 - Estimativa de fluxo sólido médio a partir de amostragens realizadas a cada 10 dias nas estações de referência do Programa HIBAM no periodo de dados comuns a todas (1998 a 1999). As amostras superficiais foram corrigidas utilizando-se de equações onde se buscou a relação entre a [MES] na seção total com a [MES] na superficie, conforme descrito em Filizola (2003).

\begin{tabular}{lcc}
\hline & $\begin{array}{c}\text { Fluxo sólido } \\
\text { mensal } \\
\text { ton.dia }\end{array}$ & $\begin{array}{c}\text { Fluxo sólido } \\
\text { interanual } \\
10^{6} \text { ton.ano }\end{array}$ \\
\hline Manacapuru, R. Solimões & 1.050 & 383 \\
\hline Paricatuba, R. Negro & 32 & 12 \\
\hline Faz. Vista Alegre, R. Madeira & 1.112 & 406 \\
\hline Óbidos, R. Amazonas & 1.669 & 609 \\
\hline Sol. + Neg. + Mad. & 2.194 & 801 \\
\hline Diferença obs. & -525 & -192 \\
\hline
\end{tabular}


observados, indicando importante fenômeno de estocagem de sedimentos na mesma porção da bacia indicada pelos dados das campanhas HIBAM, concordando mais uma vez, também, com o reportado por Meade et al. (1985). Assim, a partir desses resultados mais refinados do que aqueles das campanhas HIBAM, em termos de variação sazonal, uma taxa de sedimentação (em peso) pode ser calculada para o trecho acima referido como sendo de $19210^{6}$ ton.ano ${ }^{-1}$. Esse valor, segundo Turcq et al. (2004) e Cordeiro et al. (2008), corresponde a uma estimativa de sedimentação (vertical) variando de 0,4 a 1,35 cm.ano ${ }^{-1}$ na planície central Amazônica, notadamente nas áreas de várzea. Já para a região de antepaís (Bolívia, sobretudo), Aalto et al. (2003) indicam uma taxa de sedimentação (vertical) de 20 a $80 \mathrm{~cm}$, ocorrendo em intervalos curtos de até 8 anos, aos quais associam eventos climáticos (ENSO). Em termos de estimativa de fluxo ao oceano, esse novo conjunto de dados permite calcular um valor entre $600.10^{6}$ ton. ano $^{-1}$ (observado em Óbidos) a $800.10^{6}$ ton. ano ${ }^{-1}$ (calculado a partir dos aportes dos principais tributários), resultados que concordam sobremaneira com os de Filizola (1999) e também com a atualização daqueles dados realizada por Filizola \& Guyot (2009).

As estações hidrométricas da ANA As observações e cálculos realizados com base nos dados das estações da rede ANA (Filizola \& Guyot, 2009), atualizando Filizola (1999), mostram muita semelhança com os resultados obtidos no escopo do Programa HIBAM (dados de campanhas e dados da rede de referência), vide tabela 3. Os dados da ANA confirmam também a zona de sedimentação já destacada e existente entre Manacapuru e Óbidos e até indicam um aumento para montante (estação de Itapeuá no Rio Solimões). A área de sedimentação indicada neste estudo coincide sobremaneira com a área de baixas altitudes (entre 5 e $100 \mathrm{~m}$ de altura em relação ao nível médio do mar) detectada a partir do modelo numérico de terreno construído com dados da missão SRTM, conforme indicado por Filizola (2003) e Filizola \& Guyot (2009). Provavelmente esta grande zona de deposição se estende a jusante de

Tabela 3 - Estimativa de QS a partir de amostras coletadas na rede hidrométrica da ANA no Brasil. Fonte: Filizola \& Guyot (2009).

\begin{tabular}{lcc}
\hline & $\begin{array}{c}\text { Vazão } \\
\left(\mathrm{m}^{3} \cdot \mathrm{s}^{-1}\right)\end{array}$ & $\begin{array}{c}\text { Fluxo sólido } \\
\left(10^{6} \text { ton.ano }\right.\end{array}$
\end{tabular}

Óbidos, porém não existe nenhum dado que possa ajudar a confirmar esta hipótese até o momento. O estudo com dados da ANA também mostra a impossibilidade do uso da relação $\mathrm{QS}=\mathrm{f}(\mathrm{Q})$, para cálculo direto da descarga sólida (Filizola 1999, Filizola \& Guyot 2009). Neste caso, há interferência do sinal do pico de vazão (Q) que, como indicado pelos dados das estações de referência (Filizola 2003), não ocorre no mesmo período do pico de QS. Assim, se mostra mais adaptado o uso da relação comumente utilizada e denominada por Filizola \& Guyot (2009) como instantânea, para o cálculo de balanço, dado por:

$$
\mathrm{QS}=\mathrm{Q} \cdot[\mathrm{MES}] . \mathrm{c}
$$

No caso da equação (2), QS é a descarga sólida; [MES] é a concentração de matéria em suspensão e c é a constante para obtenção dos resultados em ton. dia $^{-1} \mathrm{e}$ é igual a 0,0864 .

Os dados da ANA, também possibilitam avaliar o percentual da carga de sedimentos produzidos na região dos escudos como sendo de $7 \%$ do total do que o Amazonas leva até os oceanos. Confirma os valores de estimativa de fluxo ao oceano dentro da mesma ordem de grandeza já citada acima, porém numa faixa um pouco abaixo daqueles valores, ou seja: 500 a $70010^{6}$ ton. ano $^{-1}$. Apesar de os dados da ANA terem por trás um método de amostragem contestado por muitos autores, que supõem que estes subestimariam os resultados, sua utilidade para estudos de fluxos de QS interanuais é válida por conta de sua grande série de dados dispersa no tempo e no espaço apresentar grande representatividade estatística. A série teve início na década de $70 \mathrm{com}$ poucas estações, a maior parte tendo iniciado as amostragens na década de 80 , com uma significativa melhora na técnica amostral a partir de meados desta década. Testes comparativos entre os métodos utilizados pelo HIBAM e pela ANA nos dias atuais foram realizados mostrando pouca discrepância em termos de resultados (Filizola \& Guyot 2004) e, por assim dizer, validando o uso daquela base de dados. Desta forma os dados da rede brasileira permitem um estudo de regularidade dos fluxos tanto líquidos quanto sólidos. Este estudo pode ser realizado através de uma metodologia simples utilizada por Jean Rodier na África (1964), indicado e adaptado por Molinier et al. (1996) para a Amazônia, porém agora com o suporte de SIG - Sistema de Informações Geográficas. Por este método, o valor da regularidade (adimensional) é obtido da razão entre as vazões médias extremas interanuais, ou seja: Qmax/Qmin (Fig. 5). O mesmo conceito foi utilizado por Filizola (2003) para os fluxos sólidos, ou seja: QSmax/QSmin. Aplicando-se os resultados às áreas de contribuição hidrológica de cada uma das estações utilizadas neste estudo, obtêm-se, para o caso das descargas líquidas, médias extremas (Fig. 6), sendo que toda a região da calha central e meio norte da Amazônia apresenta um regime bem regular no que diz respeito às suas vazões. Ou seja, as razões entre os extremos são de baixa ordem 


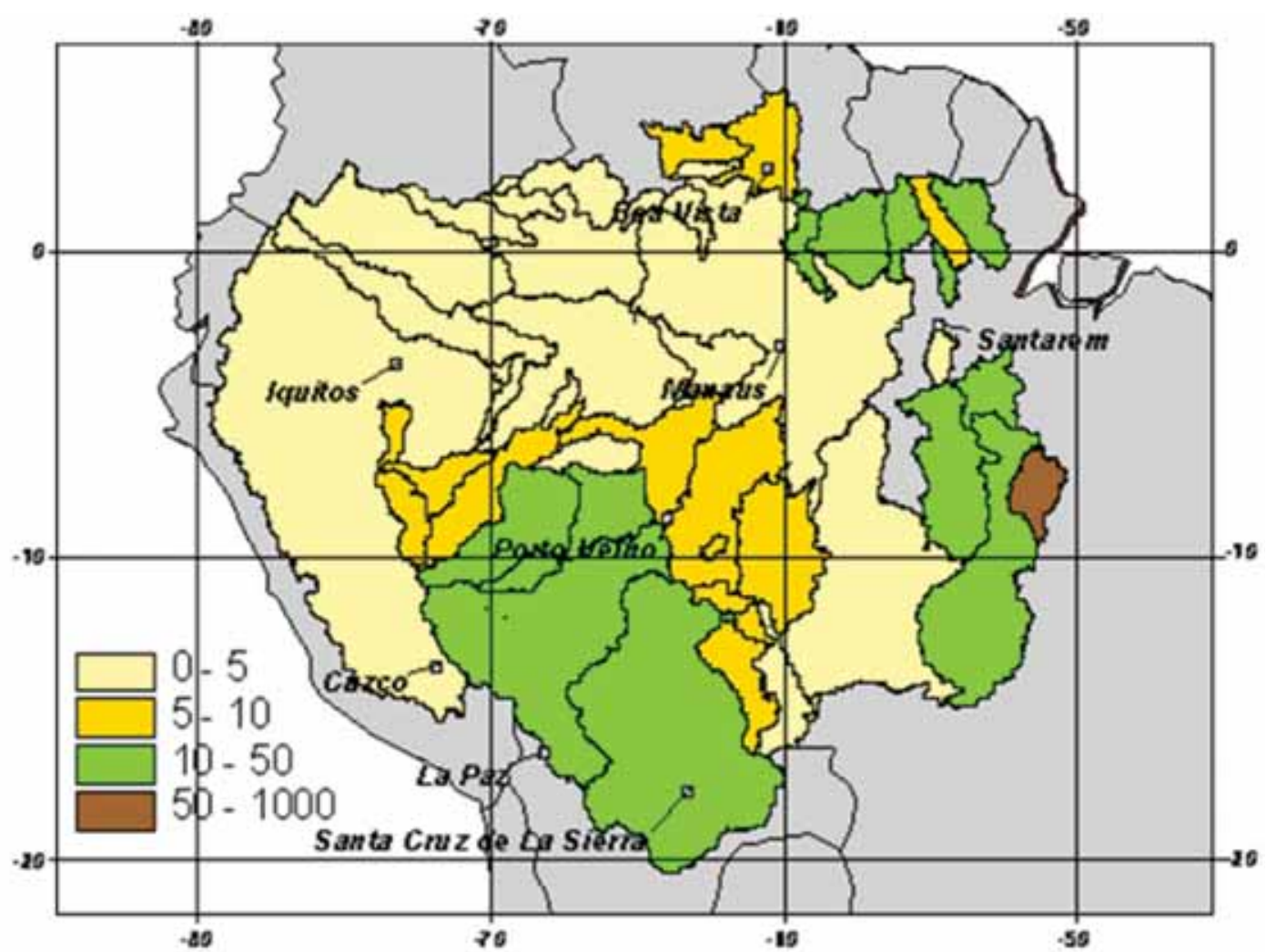

Figura 5 - Espacialização dos índices de irregularidade das vazões extremas para as "áreas de contribuição hidrológica" utilizadas no estudo. Fonte: Filizola (2003).

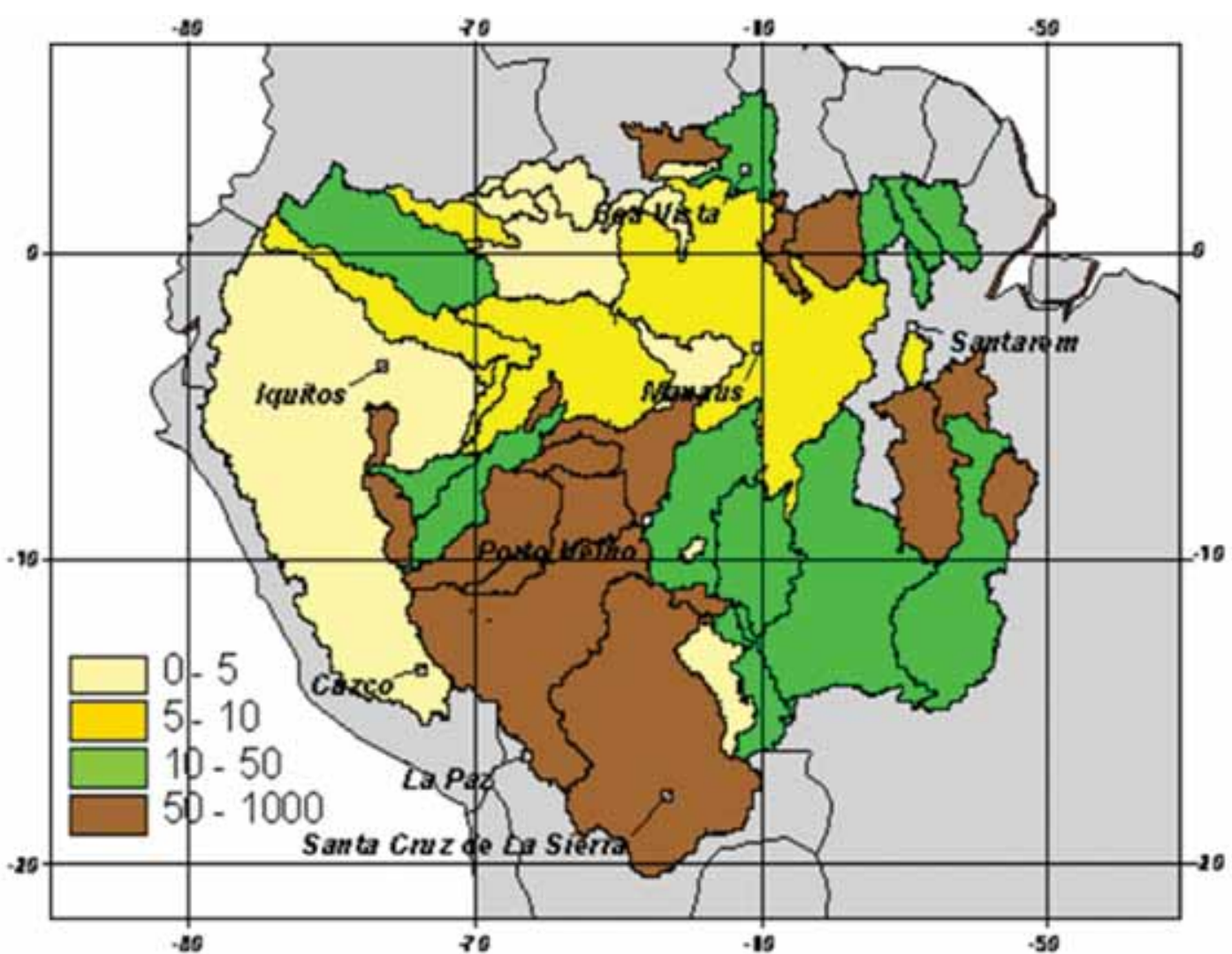

Figura 6 - Espacialização dos índices de irregularidade das descargas sólidas extremas para as "áreas de contribuição hidrológica" utilizadas no estudo. Fonte: Filizola (2003). 
(0 até 5). Os maiores valores (10 a 50) encontram-se na região sudoeste da bacia, mais especificamente na alta bacia do Rio Madeira e também na bacia do Rio Xingu (porção leste). Com relação às razões entre os extremos de QS (Fig. 6), elas indicam uma regularidade razoável na porção central (5 a 10) indo até a porção meio-norte. Para Nordeste há maior irregularidade 10 a 50 e por vezes de 50 a 1.000 . No entanto, na porção sul e sudoeste a situação é de grande irregularidade, especialmente nas bacias dos rios Purus e Madeira onde na quase totalidade os valores estão na faixa de 50 a 1000. As taxas de regularidade de QS refletem também mudanças na ordem de grandeza dos fluxos, durante o ciclo hidrológico, o que já havia sido percebido nos dados das estações de referência, quando se verificou que o Rio Madeira, em seu período de menor fluxo sólido, é superado pelas contribuições do Rio Negro, também muito baixas. No entanto, aquele rio Andino supera as contribuições do Rio Solimões e mesmo as máximas do Rio Amazonas em Óbidos, durante seu pico de máxima (meses de março e abril).

CONCLUSÕES O presente estudo procurou mostrar um resumo das atualidades em relação aos estudos sobre o fluxo de matéria em suspensão nos principais grandes rios da Bacia Amazônica realizados no escopo do Programa HIBAM, finalizado em 2001 no Brasil, e que atualmente se encontram sob uma nova perspectiva num contexto mais amplo (Pan-Amazônia) através dos estudos do consórcio internacional ORE-HYBAM (www.ore-hybam.org). Procurou mostrar a importância desses estudos quanto ao aporte de MES ao oceano do Rio Amazonas, o modo como cada grande tributário participa nesse contexto revelando a importância no aspecto regional dos rios Andinos no cômputo geral dos efeitos de sedimentação e ressuspensão identificados no seio da bacia, onde áreas podem reter anualmente até $200.10^{6}$ ton.ano ${ }^{-1}$ de material que depois entra novamente em suspensão, aparentemente por efeito do gradiente hidráulico (Meade et al. 1985). São indicadas proposições de valores de QS, quanto ao aporte do Rio Amazonas ao Oceano Atlântico como sendo da ordem de 600 a $80010^{6}$ ton.ano ${ }^{-1}$, valor que havia sido citado por Guyot et al. (2005), trabalhando apenas com dados de Óbidos. Também apresenta um hidrossedimentograma em escala mensal, obtido com dados de amostras coletadas a cada 10 dias em 10 estações de referência e que indicam uma grande importância do Rio Madeira, como sendo o responsável por modular o sinal dos fluxos líquido e sólido, tanto em escala mensal quanto anual ou interanual, além de indicar uma forte irregularidade tanto dos fluxos líquidos quanto sólidos na bacia deste rio. Ressalta-se também, as inter-relações existentes entre os diferentes tributários do Rio Amazonas e que geram em Óbidos um fluxo anual de grande monta em termos globais.

Agradecimentos Os autores agradecem aos colegas do antigo DNAEE, da CPRM, da ANEEL, da ANA e do IRD, pelo apoio na realização dos trabalhos aqui reportados em suas inúmeras fases, ao auxílio recebido do CEAP/PETROBRÁS e do Fundo Setorial de Recursos Hídricos (CR-HIDRO) quanto à infraestrutura de apoio, o que permite dar continuidade aos trabalhos aqui apresentados, ao Projeto PIATAM, à Universidade do Estado do Amazonas - UEA e à Universidade Federal do Amazonas - UFAM, parceiros que têm prestado grande auxílio nos trabalhos necessários para o funcionamento da base do Programa ORE-HYBAM em Manaus.

\section{Referências}

Aalto R., Maurice-Bourgoin L., Dunne T., Montgomery D.R., Nittrouer C., Guyot J.L. 2003. Episodic Sediment Accumulation on Amazonian Floodplains Influenced by El Niño/Southern Oscillation. Nature, 425:493-497.

Bordas M.P. 1991. An Outline of Hydrosedimentological Zones in the Brazilian Amazon Basin. In: Braga B.P.F. \& Fernandez-Jauregui C. (eds.) Water Management Of The Amazon Basin. Montevideo, Publ. Unesco-Rostlac, p. 191-203.

Bordas M.P., Lanna A.E., Semmelmann F.R. 1988. Evaluation des Risques D'érosion et de Sédimentation au Brésil à Partir de Bilans Sédimentologiques Rudimentaires. In: Bordas M.P. \& Walling D.E. (eds.) Sediment Budgets. Porto Alegre, IAHS Publ., p. 359-368.

Bouchez J., Metivier F., Lupker M., Maurice L., Perez M., Gaillardet J., France-Lanord C. 2011. Prediction of Depth-Integrated Fluxes of Suspended Sediment in the Amazon River: Particle Aggregation as a Complicating Factor. Hydrological Processes, 25(5):778-794 [doi: 10.1002/Hyp.7868].

Carvalho N.O., Filizola N., dos Santos P.M.C., Lima J.W.
2000. Guia de Práticas Sedimentométricas. Brasília, Ed. Aneel/Pnud/Omm, 154 p.

Cordeiro R.C., Turcq P.F.M., Turcq B., Moreira L.S., Rodrigues R.C., da Costa R.L., Sifeddine A., Simões Filho F.F.L. 2008. Acumulação de Carbono em Lagos Amazônicos Como Indicador de Eventos Paleoclimáticos e Antrópicos. Oecol. Bras., 12(1):116-140.

Dunne T., Mertes Lak, Meade Rh, Richey Je, Forsberg Br. 1998. Exchanges of Sediment Transport Between the Floodplain and Channel of the Amazon River in Brazil. Geological Society of America Bulletin, 110(4):450-467.

Espurt N., Baby P., Brusset S., Rodazz M., Hermoza W., Regard V., Antoine P.O. E Sala-Glasmondi. 2007. How does the Nazca Ridge Subduction Influence the Modern Amazonian Foreland Basin? Geology, 35(6):515-518 [doi: 10.1130/G23237A.1].

Ferreira J.R., Devol A.H., Martinelli L.A., Forsberg B.R., Victoria R.L., Richey J.E., Mortatti J. 1988. Chemical Composition of the Madeira River: Seasonal Trends and Total Transport. Mitt. Geol. Paläont. Inst. Univ. Hamburg, 66:63-75. 
Filizola N. \& Guyot J.L. 2009. Suspended Sediment Yield in the Amazon Basin: an Assessment Using Brazilian National Data Set. Hydrological Processes, 23(22):32073215 [doi: 10.1002/Hyp.7394].

Filizola N. 1999. O Fluxo De Sedimentos Em Suspensão Nos Rios Da Bacia Amazônica Brasileira. Brasilia, Publ. Aneel, 63 p.

Filizola N. 2003. Transfert Sédimentaire Actuel Par Les Fleuves Amazoniens. Thèse doctorat, Toulouse, Université de Toulouse III - Paul Sabatier, 273 p.

Filizola N., Sondag F., Mourão M.H., Spínola N., Cochonneau G., de Oliveira M.A., Guimarães V., Cartaxo E., Guyot J.L. 2007. Base Operacional da Rede Observacional de Parâmetros Ambientais Ore/Hybam na Amazônia Brasileira. In: Simpósio Brasileiro de Recursos Hídricos, 17, Anais.

Filizola N., Seyler F., Mourão M.H., Arruda W., Spínola N., Guyot J.G. 2009. Study of the Variability in Suspended Sediment Discharge at Manacapuru, Amazon River, Brazil. Latin American Journal of Sedimentology and Basin Analysis, 16(2):93-99.

Filizola N. \& Guyot J.L. 2004. The use of Doppler technology for suspended sediment discharge determination in the River Amazon. Hydrological Sciences-Journal des Sciences Hydrologiques, 49(1):143-153.

Gibbs R.J. 1967. The Geochemistry of the Amazon River System. Part I. The Factors that Control the Salinity and the Composition and Concentration of the Suspended Solids. Geological Society of America Bulletin, 78:12031232.

Guyot J.L. 1993. Hydrogéochimie des fleuves de l'Amazonie bolivienne. Collection Etudes \& Thèses. Paris, ORSTOM, $261 \mathrm{p}$.

Guyot J.L., Bourges J., Cortez J. 1994. Sediment Transport in the Rio Grande, an Andean River of the Bolivian Amazon Drainage Basin. In: Olive L.J., Loughran R.J., Kesby J.A. (eds.) Variability in Stream Erosion and Sediment Transport. Canberra, IAHS, p. 223-231.

Guyot J.L., Filizola N., Laraque A. 2005. Régime et bilan du flux sédimentaire de l'Amazone à Óbidos (Pará, Brésil) de 1995 à 2003. In: Walling D.E. \& Horowitz A.J. (eds.) Sediment Budgets 1. Osfordshire, IAHS, p. 347-354.

Julien P. 1995. Erosion and Sedimentation. Cambridge, Cambridge University Press, 392 p.

Meade R.H. 1985. Suspended sediment in the Amazon River and its tributaries in Brazil, during 1982-1984. Denver, U.S Geological Survey Open file Report, 39 p.

Meade R.H. 1988. Movement and storage of sediment in river systems. In: Lerman A. \& Meybeck M. (eds.) Physical and Chemical Weathering in Geochemical Cycle. Dordrecht, Kluwer, p. 165-179.

Meade R.H., Dunne T., Richey J.E., Santos U.M., Salati E. 1985. Storage and Remobilization of Suspended Sediment in the Lower Amazon River Of Brazil. Science, 228:488-490.

Meade R.H., Nordin C.F., Curtis W.F., Costa Rodrigues F.M., do Vale C.M., Edmond J.M. 1979. Sediment Loads in the Amazon River. Nature, 278:161-163.
Milliman J.D. \& Syvitzki J.P.M. 1992. Geomorphic/Tectonic Control of Sediment Discharge to the Ocean: the Importance of Small Mountainous Rivers. Journal of Geology, 100:525-544.

Molinier M., Guyot J.L., Oliveira E., Guimarães V. 1996. Les Régimes Hydrologiques de L'amazone et de ses Affluents. In: Chevallier P. \& Pouyaud B. (eds.) L'hydrologie Tropicale: Géoscience et Outil Pour le Développement. Paris, IAHS, p. 209-222.

Pinet P. \& Souriau M. 1988. Continental Erosion and Large Scale Relief. Tectonics, 7:563-582.

Rd Instruments. 1989. Acoustic Doppler Current Profilers. Principles of Operation: a Practical Primer. San Diego, Publ. Rdi, 36 p.

Richey J.E., Meade R.H., Salati E., Devol A.H., Nordin C.F., dos Santos U. 1986. Water Discharge and Suspended Sediment Concentrations in the Amazon River. Water Resources Research, 22(5):756-764.

Schmidt G.W. 1972. Amonts of Suspended Solids and Dissolved Substances in the Middle Reaches of the Amazon Over the Course of One Year (August 1969 July 1970). Amazoniana, 3(2):208-223.

Shukla J., Nobre C., Sellers P. 1990. Amazon Deforestation and Climate Change. Science, 247:1322-1325.

Sioli H. (ed.) 1984. The Amazon: Limnology and Landscape Ecology of a Mighty Tropical River and its Basin (Monographiae Biologicae). Dordrecht, Pub. Junk, 800 p.

Sioli H. 1950. Das Wasser im Amazonasgebiet. Forsch.u.Fortschr, 26:274-280.

Sioli H. 1975. Amazon tributaries and drainage basins. In: Hasler A.D. (ed.) Coupling of Land and Water Systems. New York, Springer Verlag, p. 199-213.

Sternberg H.O'r. 1975. The Amazon River of Brazil. Wiesbaden, Franz Steiner Verlag, 74 p.

Sternberg H.O'r. 1995. Waters and Wetlands of Brazilian Amazonia: an Uncertain Future. In: Nishizawa T. \& Uitto J.I. (eds.) The Fragile Tropics of Latin America: Sustainable Management of Changing Environments. Tokyo, United Nations University Press, p. 113-179.

Summerfield M.A. \& Hulton N.J. 1994. Natural Controls of Fluvial Denudation Rates in Major World Drainage Basins. Journal of Geophysical Research, 99:1387113883.

Turcq P.M., Moreira L., Cordeiro R.C., Dias G., Jouanneau J.M., Seyler P., Turcq B. (submetido). Importância dos Lagos de Várzea na Estocagem de Carbono: Várzea do Lago Grande de Curuai, Amazônia. Revista Brasileira de Geociências.

Turcq P.M., Jouanneaub J.M., Turcqc B., Seyler P., Weberb O., Guyot J.L. 2004. Carbon Sedimentation at Lago Grande de Curuai, a Floodplain Lake in the low Amazon Region: Insights into Sedimentation Rates. Palaeogeography, Palaeoclimatology, Palaeoecology, 214(1-2):27-40.

Manuscrito ID 17883 Submetido em 30 de junho de 2010 Aceito em 21 de dezembro de 2011 